\title{
RNA1-Independent Replication and GFP Expression from Tomato marchitez virus Isolate M Cloned cDNA
}

\author{
I. Ferriol, M. Turina, E. J. Zamora-Macorra, and B. W. Falk
}

First and fourth authors: Plant Pathology Department, University of California Davis, 95616, Davis; second author: Istituto per la Protezione Sostenibile delle Piante, Sez. di Torino, CNR, Turin, Italy; and third author: Colegio de Postgraduados-Campus Montecillo, 56230, Texcoco, Mexico.

Accepted for publication 4 January 2016.

\begin{abstract}
Ferriol, I., Turina, M., Zamora-Macorra, E. J., and Falk, B. W. 2016. RNA1-independent replication and GFP expression from Tomato marchitez. virus isolate $\mathrm{M}$ cloned cDNA. Phytopathology 106:500-509.

Tomato marchitez virus (ToMarV; synonymous with Tomato apex necrosis virus) is a positive-strand RNA virus in the genus Torradovirus within the family Secoviridae. ToMarV is an emergent whitefly-transmitted virus that causes important diseases in tomato (Solanum lycopersicum) in Mexico. Here, the genome sequence of the ToMarV isolate M (ToMarV-M) was determined. We engineered full-length cDNA clones of the ToMarV-M genomic RNA (RNA1 and RNA2), separately, into a binary vector. Coinfiltration of both triggered systemic infections in Nicotiana benthami-

from tomato and tomatillo plants were transmissible by the whitefly Bemisia tabaci biotype B. Also, we assessed whether these infectious clones could be used for screening tomato cultivars for resistance to ToMarV and our results allowed us to differentiate resistant and susceptible tomato lines. We demonstrated that RNA1 of ToMarV-M is required for the replication of RNA2, and it can replicate independently of RNA2. From this, ToMarV-M RNA2 was used to express the green fluorescent protein in $N$. benthamiana plants, which allowed us to track cell-to-cell movement. The construction of full-length infectious cDNA clones of ToMarV-M provides an excellent tool to investigate virus-host-vector interactions and elucidate the functions of torradovirus-encoded proteins or the mechanisms of replication of torradovirus genomic RNA.
\end{abstract} ana, tomato, and tomatillo (Physalis philadelphica) plants and recapitulated the biological activity of the wild-type virus. The viral progeny generated
Additional keywords: torradoviruses.
Tomato marchitez virus (ToMarV; synonymous with Tomato apex necrosis virus [ToANV]) is a member of the recently created genus Torradovirus (family Secoviridae). Torradoviruses have bipartite genomes consisting of two single-stranded plus-sense RNA. RNA1 is approximately $7 \mathrm{~kb}$, containing one open reading frame (ORF) encoding replication-associated proteins, including the helicase, protease, and RNA-dependent RNA polymerase (RdRp). RNA2 is approximately $5 \mathrm{~kb}$ and contains two ORF. The ORF1 in RNA2 is unique for torradoviruses, and the functions of its encoded protein are still to be elucidated. The RNA2 ORF2encoded protein has conserved regions for a putative movement protein (MP) and the three capsid proteins (CP) (Turina et al. 2007; Verbeek et al. 2008). ToMarV infections in tomato (Solanum lycopersicum) plants cause leaf necrosis and dark necrotic rings on fruit. Plants severely infected display necrosis and malformation, leading to yield reduction and substantial economic loss (Turina et al. 2007; Verbeek et al. 2008). ToMarV can be transmitted in a semipersistent manner by its whitefly vector, Bemisia tabaci biotype B (Barajas-Ortiz et al. 2013; Verbeek et al. 2014), and has only been reported in Mexico.

To date, there are only one full-length and several partial genome sequences of ToMarV (Turina et al. 2007; Verbeek et al. 2008). Little is known about the functions of torradovirus proteins or their replication and polyprotein processing strategies. Viral full-length infectious clones are essential for reverse genetic studies. Several full-length infectious clones have been generated from various

Corresponding author: B. W. Falk; E-mail address: bwfalk@ucdavis.edu

*The $\boldsymbol{e}$-Xtra logo stands for "electronic extra" and indicates that five supplementary figures and two supplementary tables are published online.

http://dx.doi.org/10.1094/PHYTO-10-15-0267-R

(C) 2016 The American Phytopathological Society species of the family Secoviridae (Atsumi et al. 2013; Komatsu et al. 2013) but, until now, infectious clones of only one virus of the genus Torradovirus, Tomato torrado virus (ToTV), have been developed (Wieczorek et al. 2015).

Here, we determined the complete nucleotide sequences for ToMarV isolate M (ToMarV-M) genomic RNA (gRNA)1 and -2 and developed an agroinoculation system that can trigger an infection in Nicotiana benthamiana, tomato, and tomatillo (Physalis philadelphica) plants. We confirmed the biological activity of ToMarV-M cDNA clones including, for the first time for torradoviruses, whitefly transmission of progeny virus, and used this system to demonstrate ToMarV-M resistance and susceptibility in tomato. Also, we engineered a ToMarV-M RNA2 cDNA clone to express the green fluorescent protein (GFP) in $N$. benthamiana plants by inserting the GFP gene between the predicted MP and VP35 CP coding regions of ToMarV-M. Finally, we demonstrated, for the first time for torradoviruses, that RNA1 of ToMarV-M is required for the replication of RNA2 but can replicate independently from RNA2. These tools will allow us to elucidate the role of viral encoded proteins in the infection cycle of ToMarV.

\section{MATERIALS AND METHODS}

Virus source and RNA extraction. ToMarV-M was collected in a survey in 2005 of field tomatoes in Sinaloa, Mexico (Turina et al. 2007). ToMarV-M was maintained in tomatillo and N. benthamiana plants in a greenhouse in the University of California-Davis BSL 3P Contained Research Facility under conditions of $16 \mathrm{~h}$ of light and $8 \mathrm{~h}$ of darkness at $24^{\circ} \mathrm{C}$. Total RNA was extracted from $100 \mathrm{mg}$ of leaf tissue using TRIzol Reagent (Life Technologies, Carlsbad, CA) or a Spectrum Plant Total RNA kit (Sigma-Aldrich, St. Louis), following the manufacturer's instructions. The RNA was resuspended in $25 \mu \mathrm{l}$ of diethyl pyrocarbonate-treated distilled water. 
gRNA was extracted from ToMarV-M virions using the RNeasy Mini Kit (Qiagen, Germantown, MD), following the manufacturer's instructions.

Sequencing of ToMarV-M. The full-length sequences of RNA1 and RNA2 of ToMarV-M were obtained by one-step reversetranscription polymerase chain reaction (RT-PCR) using overlapping primers that covered ToMarV-M RNA1 and RNA2 nucleotide sequences. All primers are described in Supplementary Table S1. Primers were designed based on the sequence of ToMarV isolate PRI-TMarV0601 (EF681764 and EF681765) and ToANV isolate VE434 (EF063641 and EF063642). One-step RT-PCR was performed in $25 \mu \mathrm{l}$ of reaction mix containing $5 \mu \mathrm{l}$ of GoTaq Flexi DNA polymerase mix (Promega Corp., Madison, WI), $0.5 \mu \mathrm{M}$ each forward and reverse primer, $1.25 \mu \mathrm{l}$ of $0.1 \mathrm{M}$ dithiothreitol and $0.2 \mathrm{mM}$ dNTP mix, $8 \mathrm{U}$ of SuperScript II reverse transcription (Life Technologies), and $0.65 \mathrm{U}$ of GoTaq Flexi (Promega Corp.). Cycling conditions included an initial incubation at $42^{\circ} \mathrm{C}$ for $60 \mathrm{~min}$; followed by $2 \mathrm{~min}$ at $94^{\circ} \mathrm{C} ; 40$ cycles of $94^{\circ} \mathrm{C}$ for $15 \mathrm{~s}, 55^{\circ} \mathrm{C}$ for $20 \mathrm{~s}$, and $72^{\circ} \mathrm{C}$ for $1 \mathrm{~min} 30 \mathrm{~s}$; and a final incubation at $72^{\circ} \mathrm{C}$ for $10 \mathrm{~min}$. The amplified products were visualized on $2 \%$ agarose/Tris-acetate-EDTA gel stained with SYBR Safe DNA Gel Stain (Life Technologies) and purified with the MinElute Gel Extraction kit (Qiagen). The nucleotide sequences of the 5' and 3' ends of each RNA were determined using the 5' -rapid amplification of cDNA ends (RACE) and 3'-RACE systems (Life Technologies), according to the manufacturer's instructions. Specific primers were used for each RNA. Nucleotide sequences were obtained with the Sanger sequencing method and assembled with the program STADEN 2.0.0b9 (Bonfield et al. 1995). Full-length nucleotide sequences of RNA1 and RNA 2 were deposited in GenBank under accession numbers KT756874 to KT756875.

Construction of full-length genome cDNA clones. The full-length cDNA clones were constructed with two overlapping cDNA fragments spanning the complete ToMarV-M RNA1 and RNA2 that were first cloned into pGEMT-Easy. First, the DNA fragments were obtained by RT-PCR using total RNA from ToMarV-M-infected tomatillo plants and primers shown in Supplementary Table S2. RT was performed with the SuperScript III First-Strand Synthesis System (Life Technologies) and the primers ToANVR1.R and ToANVR2.R. RNA1 and RNA2 were amplified using the primers pG-R1-A.F/pG-R1-A.R and pG-R1-B.F/pG-R1B.R for RNA1 and pG-R2-C.F/pG-R2-C.R and the primers pG-R2D.F/pG-R2-D.R for RNA2. PCR was carried out using the iProof High-Fidelity PCR Kit (Bio-Rad, Hercules, CA). The DNA products were gel purified, ligated into pGEMT-Easy (Promega Corp.), and transformed into Escherichia coli DH5o-competent cells. The insert orientations were confirmed using restriction enzyme digestion. The full-length RNA2 was successfully cloned into pGEMT-Easy using the restriction enzymes BamHI and NdeI (data not shown). The resulting plasmids (pGEMT-Easy-R1-A, pGEMT-Easy-R1-B, and pGEMT-Easy-R2-C+D) were used to clone RNA1 and RNA2 into the binary vector pJL89 (Wang et al. 2009) (Supplementary Fig. S1). PCR was carried out using CloneAmp HiFi PCR Premix (Clontech, Mountain View, CA). In-Fusion reaction and transformation was performed using In-Fusion HD Cloning Kit (Clontech) and Stellar Competent cells (Clontech). The presence of the full-length ToMarV-M RNA1 and RNA2 was confirmed by restriction enzyme digestion and the resulting plasmids were named pJL89-M-R1 and pJL89-M-R2 (Fig. 1). Nucleotide sequences were obtained by Sanger sequencing and assembled with the program STADEN 2.0.0b9 (Bonfield et al. 1995). Full-length nucleotide sequences of pJL89M-R1 and pJL89-M-R2 were deposited in GenBank under accession numbers KT756876 to KT756877.

Sequence analyses. BLASTX and BLASTN analyses were performed to compare the nucleotide and amino acid sequences of ToMarV-M with the available sequences in GenBank (Altschul et al. 1990). Nucleotide alignment of ToMarV-M and pJL89-M-R1 and pJL89-M-R2 sequences was performed using CLUSTALW implemented in the software MEGA6 (Tamura et al. 2013).

Engineering ToMarV-M RNA-2 to express GFP in plants. RNA2 of ToMarV-M was engineered to express GFP by inserting the GFP coding sequence between the putative MP and VP35 CP predicted coding regions (Fig. 1B). The GFP coding region was amplified using pCT74 (Lorang et al. 2001) and the primers GFP.F/GFP.R, and pJL89-M-R2 was linearized by PCR using Vector.F/Vector.R. In-Fusion reaction and transformation was performed using the In-Fusion HD Cloning kit and Stellar Competent cells. The resulting plasmid, pJL89-M-R2-GFP, had the putative cleavage site between the putative MP and VP35 CP duplicated at the $\mathrm{N}$-terminal and also at the $\mathrm{C}$-terminal region of the GFP (Fig. 1B). The presence of the GFP gene in the construct pJL89-M-R2-GFP was confirmed by sequencing.

Agroinfiltration assays. Agroinoculation experiments were carried out in plants of $N$. benthamiana (four- to six-leaf stage), tomato line HMC101 (two fully expanded cotyledons and two emerging small true leaf stage), and tomatillo (two fully expanded cotyledons and two emerging small true leaf stage). Plants were grown under conditions of $16 \mathrm{~h}$ of light and $8 \mathrm{~h}$ of darkness at $24^{\circ} \mathrm{C}$. Plasmids were transformed into Agrobacterium tumefaciens strain $\mathrm{C} 58 \mathrm{C} 1$ and agroinfiltrated as previously described (Wang et al. 2009). Equal volumes of A. tumefaciens cells containing pJL89-M-R1 and pJL89-M-R2 were mixed prior to infiltration. For the experiments with pJL89-M-R2-GFP, A. tumefaciens suspensions diluted at an optical density (OD) at $600 \mathrm{~nm}$ of 0.4 were agroinfiltrated following a serial 10-fold dilution from 1 to 1:100 (for Western blot experiments) and 1:5 to 1:5,000 (for microscopy experiments), and coagroinfiltrated with pJL89-M-R1 and pBIN-P19, a binary vector constitutively expressing the gene silencing suppressor of Tomato bushy stunt virus (TBSV) p19. We used pBIN-GFP (Crivelli et al. 2011) using a bacterial culture at the same original OD as that of the pJL89-M-R2-GFP in the same pJL89-M-R1, plus pBIN-P19 mix as a control.

To exclude the possibility that GFP can move cell to cell independently from the virus, a further dilution series of A. tumefaciens cells expressing pBIN-GFP was done in a mix at $\mathrm{OD}=0.4$ of wildtype RNA1- and RNA2-expressing agrobacteria.

Western blot and Northern blot analyses. Western blot analyses were carried out as previously described (Wang et al. 2009). To detect ToMarV-M CP, the blots were probed using a 1:2,000 dilution of anti-ToANV-virion as the primary antibody (Turina et al. 2007). Goat antirabbit immunoglobulin $\mathrm{G}$ (IgG) (H+L)-AP or goat antirabbit IgG-HRP conjugate (Bio-Rad) at a 1:2,000 dilution were used as a secondary antibody. To detect GFP, the blots were probed using a 1:500 dilution of anti-GFP-HRP conjugated antibody (Santa Cruz Biotechnology, Dallas, TX). The nitrocellulose membranes were developed using the AP Conjugate Substrate kit (Bio-Rad) or SuperSignal West Pico Chemiluminescent Substrate (Thermo Fisher Scientific, Waltham, MA). Proteins were imaged with the ChemiDoc Touch Imaging System (Bio-Rad) and analyzed with the software Image Lab 5.2.1 (Bio-Rad). Northern blot analyses were carried out as previously described (Wang et al. 2009). Briefly, negative- and positive-strand probes were derived by cloning fragments of each RNA in pGEMT-Easy (Promega Corp.). A probe for RNA1 was derived with a 327-bp fragment (positions 77 to 403 in RNA1) obtained through PCR by using primers RNA1-Probe.F/RNA1Probe.R. The PCR product was cloned into pGEMT-Easy (Promega Corp.). Both orientations were kept and linearized for transcription with EcoICRI. Similarly, a probe for RNA2 (348-bp fragment, positions 42 to 389 in RNA2) was obtained by using the primers RNA2-Probe.F/RNA2-Probe.R.

Virion purification, RNA and protein analysis, and transmission electron microscopy. Virions were purified from $200 \mathrm{~g}$ of ToMarV-M-infected $N$. benthamiana plants, as previously described (Turina et al. 2007). The presence of virions was determined by sodium dodecyl sulfate polyacrylamide gel electrophoresis and Coomassie Brilliant Blue R-250 staining (Sambrook and Russell David 1989) 
(data not shown). Purified virion suspension $(10 \mu \mathrm{l})$ was stained with $2 \%$ uranyl acetate and bacitracin at $0.01 \mathrm{mg} / \mathrm{ml}$ and examined for virus particles with a transmission electron microscope (Hitachi H-7500; Chiyoda, Tokyo). The presence of gRNA in the ToMarV-M virions was confirmed by loading $200 \mathrm{ng}$ of gRNA in an RNase-free $1.2 \%$ agarose HEPES-EDTA (0.02 M HEPES, 0.001 MEDTA, and 0.003 M $\mathrm{NaOH}, \mathrm{pH}$ 7) denaturing gel, then staining it with ethidium bromide. Immunosorbent electron microscopy (ISEM) was carried out using sap from agroinfiltrated leaves 2 and 3 days postagroinfiltration (dpa) as previously described (Rossi et al. 2015) but using anti-
ToANV-virion antibodies at a 1:1,000 dilution absorbed onto Formvar and carbon-coated grids.

Whitefly transmission experiments. Nonviruliferous $B$. tabaci biotype $\mathrm{B}$ whiteflies were transferred to two tomatillo and tomato plants infected by agroinfiltration with pJL89-M-R1 and pJL89M-R2 by gentle shaking from several cotton leaves. Plants were kept in a Conviron chamber (Conviron, Temecula, CA) under $16 \mathrm{~h}$ of light and $8 \mathrm{~h}$ of darkness at $24^{\circ} \mathrm{C}$. Infected plants were removed after an acquisition access period (AAP) of $24 \mathrm{~h}$. Four healthy tomato and four healthy tomatillo plants were used as receptor

A

RNA1 (7,226 nt)

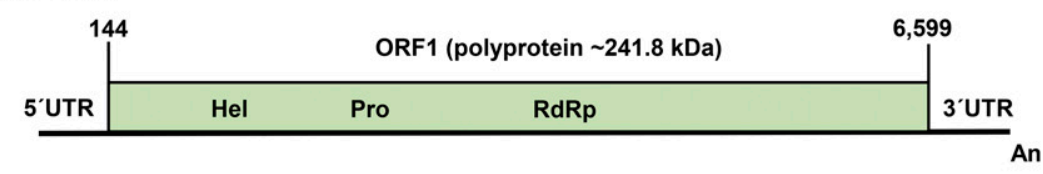

RNA2 (4,897 nt)

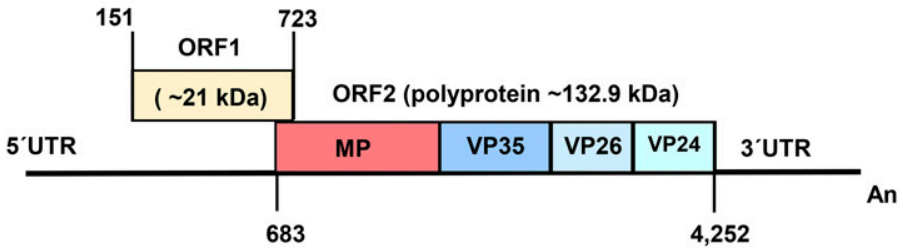

B

pJL89-M-R1

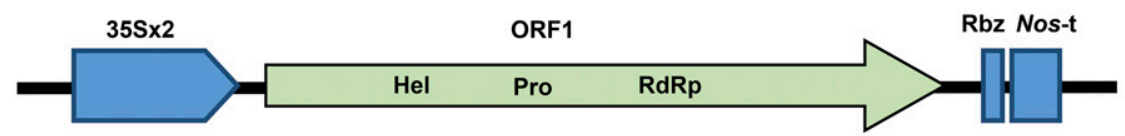

pJL89-M-R2

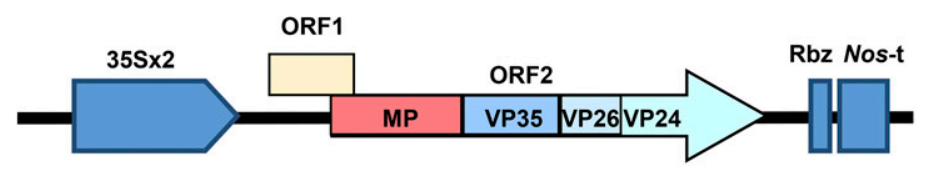

pJL89-M-R2-GFP

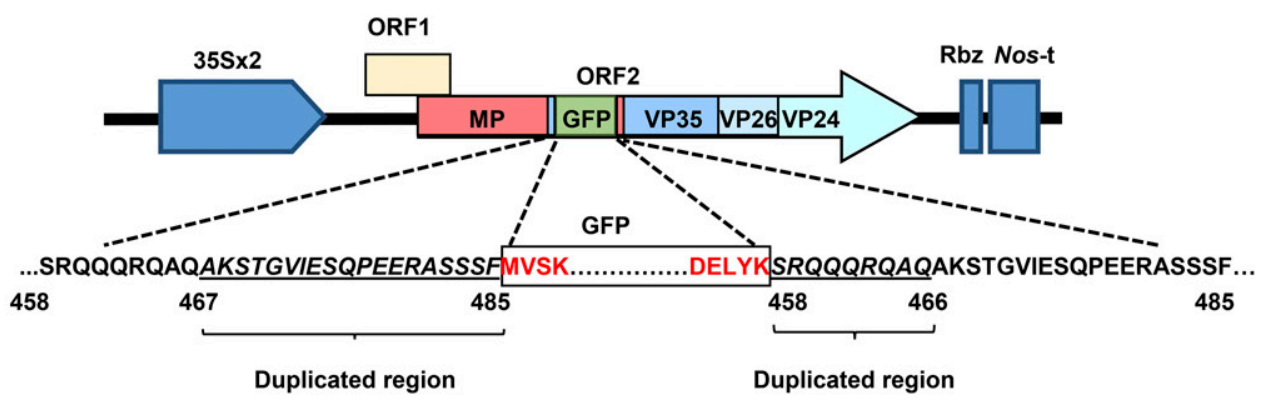

Fig. 1. Genome organization of Tomato marchitez virus isolate M (ToMarV-M) and constructs used in this study. A, Genome organization of ToMarV-M. The RNA genome is depicted with horizontal lines. The polyadenylated tail is shown at the $3^{\prime}$ end of the RNA (An). The boxes indicate open reading frames (ORF) and the relative positions of regions containing motifs of helicase (Hel); 3C-like protease (Pro); RNA-dependent RNA polymerase (RdRp); putative movement protein (MP); and capsid proteins VP35, VP26, and VP24. Nucleotide positions corresponding to initiation and termination codons are shown. The 5' and 3' untranslated regions (UTR) are shown. B, Constructs used in this study. pJL89-M-R1 and pJL89-M-R2 comprise the full genomes of RNA1 and RNA2, respectively. The duplicated $35 \mathrm{~S}$ promoter $(35 \mathrm{~S} \times 2)$ of the Cauliflower mosaic virus is immediately upstream of the first nucleotide of both cDNA. The Hepatitis delta virus ribozyme $(\mathrm{Rbz})$ and the nopaline synthase terminator (Nos- $\mathrm{t}$ ) are engineered in tandem after a poly A of 25 As. Plasmid pJL89-M-R2-GFP comprises the full genome of RNA2 and the green fluorescent protein (GFP) coding sequence inserted between the predicted MP and the VP35 capsid protein coding regions of ToMarV-M. Duplicated amino acids between the MP and the VP35 capsid protein of ToMarV-M are underlined and in italics. The GFP N-terminal and C-terminal amino acid sequence is indicated with a red box. 
plants for each group of whiteflies (approximately 100 per plant) coming from a single acquisition plant. After an inoculation access period (IAP) of $72 \mathrm{~h}$, the plants were sprayed with insecticide and transferred to a greenhouse under conditions of $16 \mathrm{~h}$ of light and $8 \mathrm{~h}$ of darkness at $24^{\circ} \mathrm{C}$. Symptoms were scored 2 to 3 weeks postinoculation.

Screening of ToMarV-M-resistant and -susceptible tomato cultivars. The tomato lines HMC101, HMC102, and HMC103 were tested for resistance to ToMarV-M using agroinfiltration and whitefly transmission. Cotyledons of nine tomato plants of each line were agroinfiltrated with an equal mix of the cDNA clones pJL89M-R1 and pJL89-M-R2. A transmission assay was carried out using ToMarV-M-infected tomatillo plants as source plants, $24 \mathrm{~h}$ of AAP, and $72 \mathrm{~h}$ of IAP. Ten tomato plants of each line were used as receptor plants.

GFP expression in $N$. benthamiana plants. Plant tissue from $N$. benthamiana plants agroinfiltrated with the combination of pJL89-M-R1 and pBIN-P19 and serial dilutions of pJL89-M-R2GFP were examined for GFP fluorescence. Plants were examined at
24, 48, and $72 \mathrm{~h}$ postagroinfiltration. Plants agroinfiltrated with pBIN-GFP (Crivelli et al. 2011) in combination with pBIN-P19 and pJL89-M-R1 were used as a positive control. Examination of fluorescence was performed using a Leica TCS-SP2 microscope (Leica Microsystems, Wetzlar, Germany). Images were captured using a $\times 10$ or $\times 20$ objective. A 488-nm Argon laser line was used to excite GFP, which was imaged using a collection window of 500 to $525 \mathrm{~nm}$. Pictures were processed (for trimming, adjustment of contrast and brightness, and figure assembly) using the GNU Image Manipulation Program (https://www.gimp.org/).

\section{RESULTS}

Complete nucleotide sequences of ToMarV-M isolate $M$ RNA1 and -2. ToMarV-M full-length genome sequences have been determined. RNA1 has 7,226 nucleotides (nt), excluding the polyadenylated 3' end (Fig. 1A). A BLASTN analyses with the nucleotide sequence of ToMarV-M (KT756874) RNA1 revealed 99 and $95 \%$ nucleotide identity with RNA1 sequences of ToANV

A
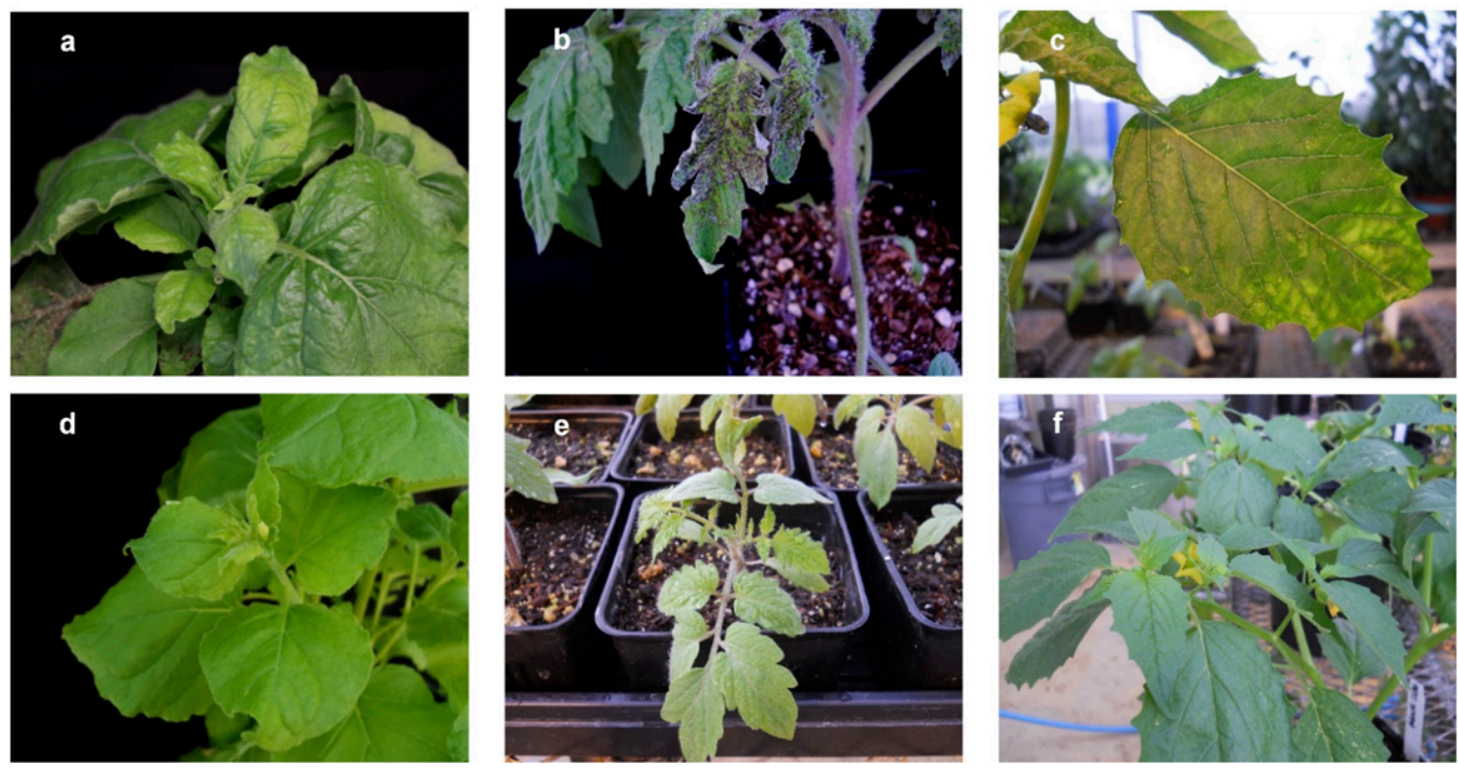

B

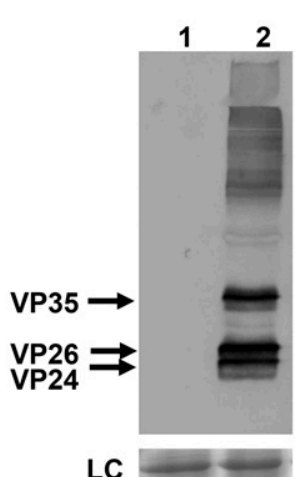

C

D $\begin{array}{lll}1 & 2 & 3\end{array}$
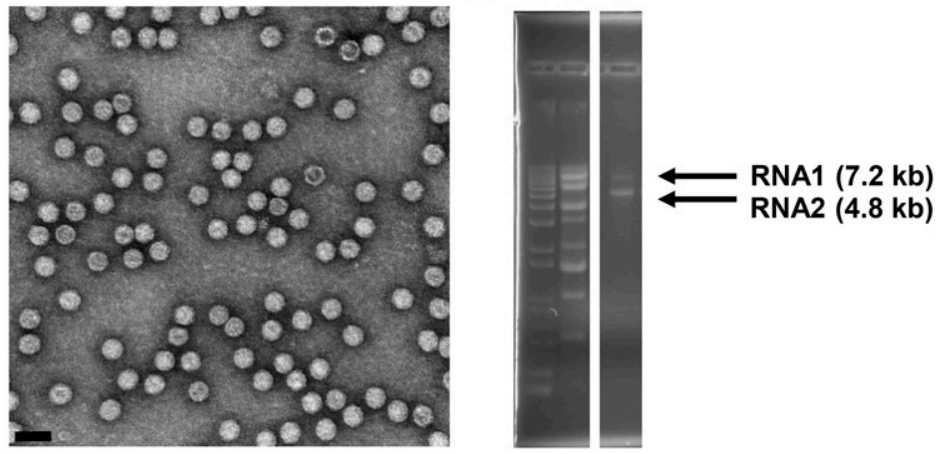

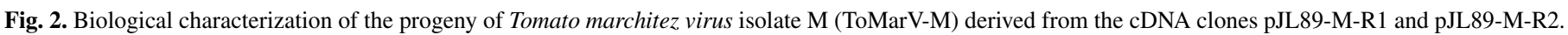

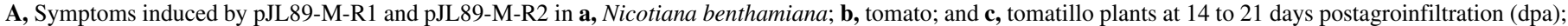

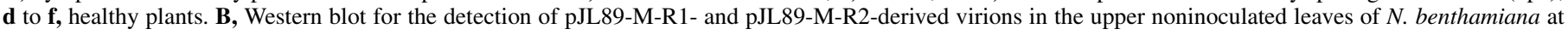

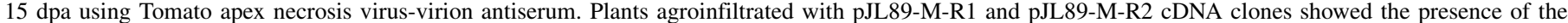

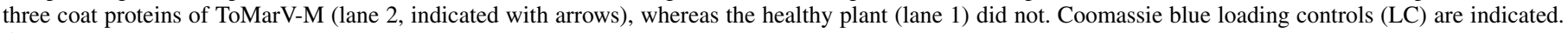

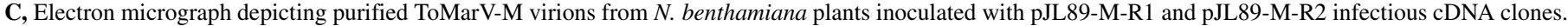

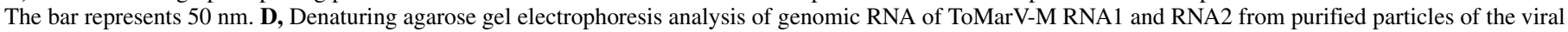

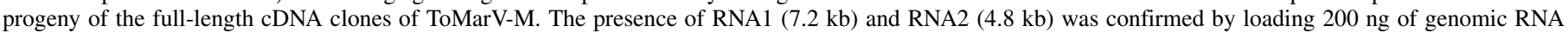

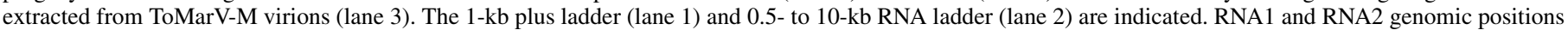
are indicated with arrows. 
isolate VE434 (EF063641) and ToMarV isolate PRI-TMarV0601 (EF681764), respectively. RNA1 has one ORF of 2,151 amino acids (aa), which codes for a predicted polyprotein with a molecular weight of approximately $241.8 \mathrm{kDa}$. Analysis of the ORF1 polyprotein revealed domains having similarity with helicase, protease, and RdRp motifs (Fig. 1A). The amino acid sequence of the RNA1 ORF1 polyprotein is $99 \%$ identical to ToANV isolate VE434 (EF063641) and ToMarV isolate PRI-TMarV0601 (EF681764). The 5' untranslated region (UTR) of RNA1 has $143 \mathrm{nt}$ and begins with UUUAAAAG (Supplementary Fig. S2) whereas the 3' UTR of RNA1 has 627 th, excluding the poly A tail. A BLASTN analysis with ToMarV-M RNA1 5' UTR (KT756874) revealed 96\% nucleotide identity with ToMarV isolate PRI-TMarV0601 (EF681764). BLASTN analysis with ToMarV-M RNA1 3' UTR (KT756874) revealed 99 and $90 \%$ nucleotide identity with ToANV isolate VE434 (EF063641) and ToMarV isolate PRI-TMarV0601 (EF681764), respectively.

RNA2 has 4,897 nt, excluding the polyadenylated 3' end (Fig. 1A). A BLASTN analysis with ToMarV-M (KT756875) RNA2 revealed 98 and $80 \%$ nucleotide identity with ToANV isolate VE434 (EF063642) and ToMarV isolate PRI-TMarV0601 (EF681765), respectively. RNA2 has two partially overlapping ORFs. ORF1 encodes 190 aa, which gives a putative protein with a molecular weight of approximately $21.1 \mathrm{kDa}$. BLASTX analysis indicated that ORF1 has $85 \%$ amino acid identity with ToMarV isolate PRI-TMarV0601 (EF681765). ORF2 encodes a protein of 1,189 aa, with a molecular weight of approximately $132.9 \mathrm{kDa}$. The amino acid sequence of the RNA2 ORF2 polyprotein is 89 and $99 \%$ identical to ToMarV isolate PRI-TMarV0601 (EF681765) and ToANV isolate VE434 (EF063642), respectively. The 5' UTR of RNA2 has 150 nt and begins with UUUAAAAG whereas the $3^{\prime}$ UTR of RNA2 has $645 \mathrm{nt}$, excluding the poly A tail. A BLASTN analyses with ToMarV-M RNA2 5' UTR revealed 88\% nucleotide identity with ToMarV isolate PRI-TMarV0601 (EF681765).
BLASTN analyses with ToMarV-M RNA2 3' UTR showed 92 and 90\% nucleotide identity with ToMarV isolate PRI-TMarV0601 (EF681765) and ToANV isolate VE434 (EF063642), respectively.

Construction of full-length cDNA clones of ToMarV-M. To construct full-length cDNA clones of ToMarV-M, two cDNA fragments of RNA1 and two of RNA2 of the isolate ToMarV-M were amplified and assembled in pGEMT-Easy. We first attempted to join the two overlapping fragments of ToMarV-M RNA1 into pGEMT-Easy. However, repeated attempts to transform into E. coli DH5 $\alpha$ with the full-length RNA1 yielded clones with inserts shorter than expected (data not shown). By contrast, the full-length RNA2 was successfully cloned into pGEMT-Easy using the restriction enzymes BamHI (unique to ToMarV-M RNA2 genome) and NdeI (unique to pGEMT-Easy). To obtain infectious clones suitable for agroinfiltration, the full-length ToMarV-M cDNA were placed between the Cauliflower mosaic virus $35 \mathrm{~S}$ promoter and the Hepatitis delta virus ribozyme followed by the Nos- $t$ terminator in the binary vector pJL89. A poly (T) tail of $25 \mathrm{nt}$ was added at the end of the cDNA of each genomic segment. To minimize the presence of nonviral residues at the $5^{\prime}$ end of the viral progeny, the cDNA was inserted immediately following the $35 \mathrm{~S}$ promoter. RNA1 and RNA2 cDNAs were cloned into pJL89 using the In-Fusion HD Cloning kit, as described above. The pJL89-M-R1 and pJL89-M-R2 cDNAwere sequenced using overlapping primers covering the full-length genome and deposited in the GenBank under accession numbers KT756876 and KT756877. Sequence analysis showed differences between ToMarV-M (KT756874 and KT756875) and the full-length cDNA clones (KT756876 and KT756877). RNA1 has five nucleotide differences (one nonsynonymous, resulting in amino acid substitution K705R) with respect to the sequence of ToMarV-M. RNA2 has six nucleotide differences with respect to the sequence of ToMarV-M (one nonsynonymous in ORF2, resulting in amino acid substitution V602I).
A

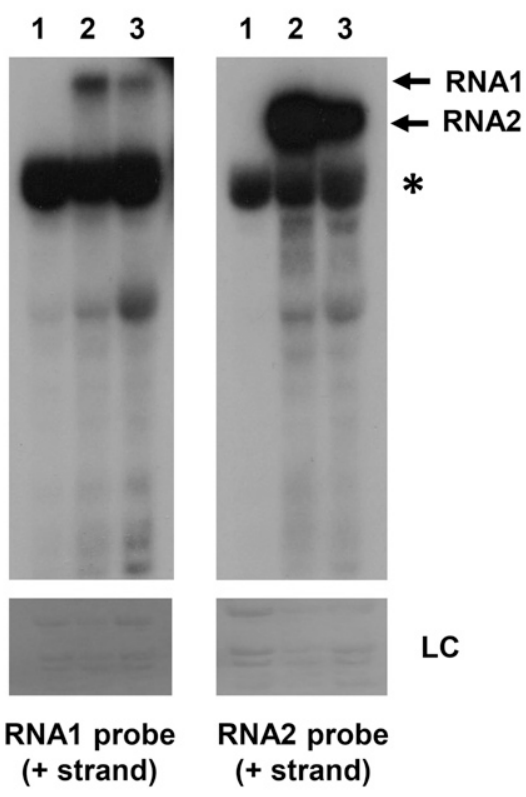

B

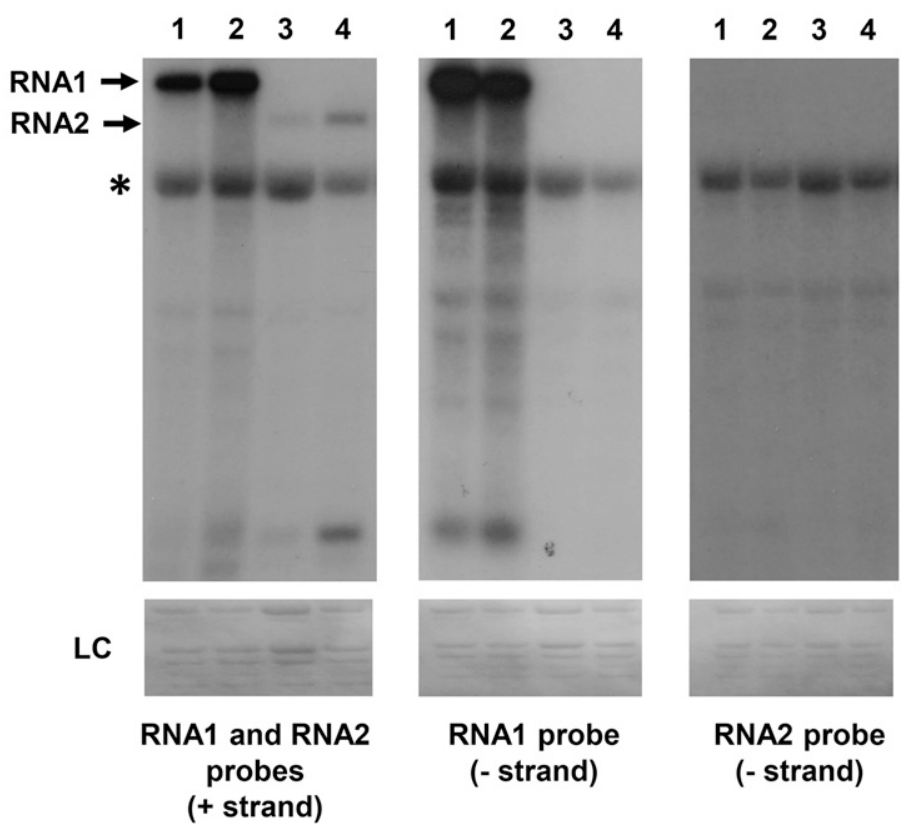

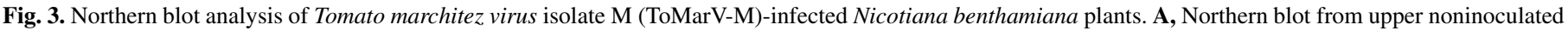

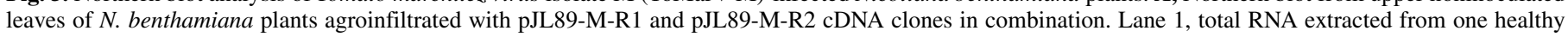

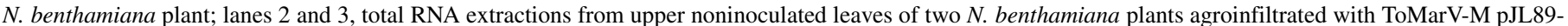

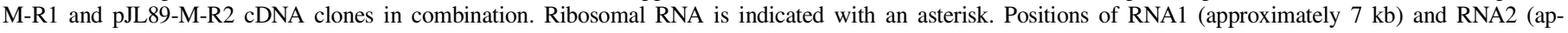

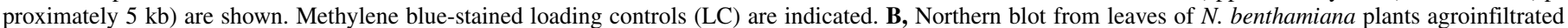

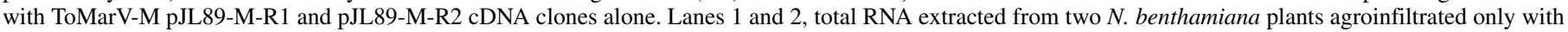

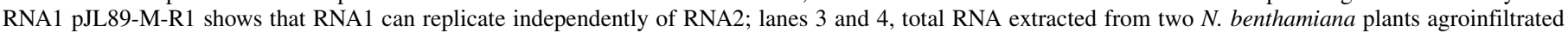

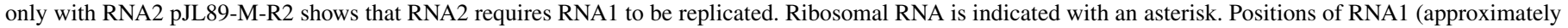
$7 \mathrm{~kb}$ ) and RNA2 (approximately $5 \mathrm{~kb}$ ) are indicated. Methylene blue LC are indicated. 
ToMarV-M full-length cDNA clones are infectious in $N$. benthamiana, tomatillo, and tomato plants. To test their infectivity, pJL89-M-R1 and pJL89-M-R2 were transformed into A. tumefaciens strain $\mathrm{C} 58 \mathrm{C} 1$. Then, cells were coinfiltrated into leaves of three host species: tomato line HMC101 (S. lycopersicum L.), tomatillo ( $P$. philadelphica), and $N$. benthamiana. Tomato plants began to show symptoms 5 dpa and, after 2 weeks, all plants showed symptoms indistinguishable from that of the wild-type ToMarV-M infection (Fig. 2A). The results showed that pJL89-M-R1 and pJL89-M-R2 gave rise to systemic infections in the three plant hosts and this was confirmed by Western blot using ToANV-virion antibodies (data not shown). Agroinfiltration experiments were repeated more than three independent times. Western blots of $N$. benthamiana plants agroinfiltrated with ToMarV-M cDNA clones confirmed the presence of ToMarV-M in the apical leaves (Fig. 2B).

$N$. benthamiana plants were agroinfiltrated with pJL89-M-R1 and

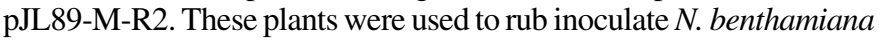
and obtain purified virions. These virions $(10 \mu \mathrm{l})$ were then rub inoculated to $N$. benthamiana plants, and the inoculated plants showed typical ToMarV-M symptoms 14 days postinoculation. Purified ToMarV-M virions were also examined by transmission electron microscopy (TEM), revealing the presence of spherical particles of about $30 \mathrm{~nm}$ in diameter (Fig. 2C). The dimensions and morphology of these particles were indistinguishable from those of ToMarV isolate PRI-TMarV0601 and ToANV isolate VE434 (Turina et al. 2007; Verbeek et al. 2008). RNA was extracted from purified virions and analyzed by agarose gel electrophoresis. The results showed the presence of two RNAs of approximately 7.2 and $4.8 \mathrm{~kb}$, as previously described (Fig. 2D) (Turina et al. 2007).

ToMarV-M RNA1 can replicate independently of RNA2 and is required for the replication of RNA2. RNA1 genome segments for many members of the family Secoviridae can replicate independently from RNA2 (Goldbach et al. 1980; Robinson et al. 1980) but this has not been tested for any member of the genus Torradovirus. Therefore, we used pJL89-M-R1 and pJL89-M-R2 cDNA clones individually and in combination to test this hypothesis. Northern blots of $N$. benthamiana plants agroinfiltrated with pJL89M-R1 and pJL89-M-R2 cDNA clones confirmed the presence of ToMarV-M in the apical leaves (Fig. 3A). Next, the accumulation of each gRNA alone was evaluated in the agroinfiltrated areas through Northern blot. Specific positive- and negative-strand RNA1 and RNA2 probes were used (Fig. 3B). Agroinfiltration of both gRNA pJL89-M-R1 and pJL89-M-R2 was performed in combination with the silencing suppressor pBIN-P19 from TBSV. When we infiltrated $N$. benthamiana leaves with pJL89-M-R1 alone, Northern blot analysis of infiltrated leaves showed accumulation of both negative- and positive-strand ToMarV-M RNA1, demonstrating that ToMarV-M RNA1 can replicate independently of RNA2 (Fig. 3B). However, when pJL89-M-R2 was infiltrated without the presence of RNA1, only accumulation of positive-strand RNA2 resulting from $35 \mathrm{~S}$ promoter transcription was detected by Northern blot. This result demonstrated that RNA1 is required for the replication of RNA2 of ToMarV-M. When pJL89-M-R1 and pJL89-M-R2 were mixed, both positive- and negative-strand RNA were detected for both RNA.

Engineering ToMarV-M RNA-2 to express GFP in plants. The GFP gene was inserted in the RNA2 ORF2 between the putative $\mathrm{MP}$ and the VP35 CP coding regions to assess the expression of GFP in $N$. benthamiana (Fig. 1B). The cleavage site between the MP and VP35 CP of ToMarV-M has not yet been determined. Nevertheless, the cleavage site between the MP and VP35 in ToTV occurs upstream of amino acid position 486 of RNA2 ORF2 (Verbeek et al. 2007). To duplicate the putative cleavage site between the MP and VP35 of ToMarV-M, we inserted the GFP coding sequence after amino acid position 485 of the RNA2 ORF2 encoded polyprotein of ToMarV-M (KT756875). We duplicated a region of 19 aa (positions 467 to 485, KT756875) before the N-terminal region of the GFP gene. Moreover, 9 aa (positions 458 to $466, \mathrm{KT} 756875$ ) were duplicated after the C-terminal region of the GFP gene (Fig. 1B). To prevent homologous recombination and removal of the GFP gene, the nucleotide sequence of the duplicated regions were changed but the encoded amino acid residues were unchanged (Fig. 1B). $N$. benthamiana plants were agroinfiltrated with pJL89-M-R2-GFP in combination with RNA1 pJL89-M-R1 and pBIN-P19. Western blot analysis demonstrated the accumulation of GFP and ToMarV-M CP 3 dpa (Fig. 4A). A protein reacting with GFP antibodies and migrating at the same position as GFP expressed from pBIN-GFP was detected from the area

\section{A} GFP Antibody

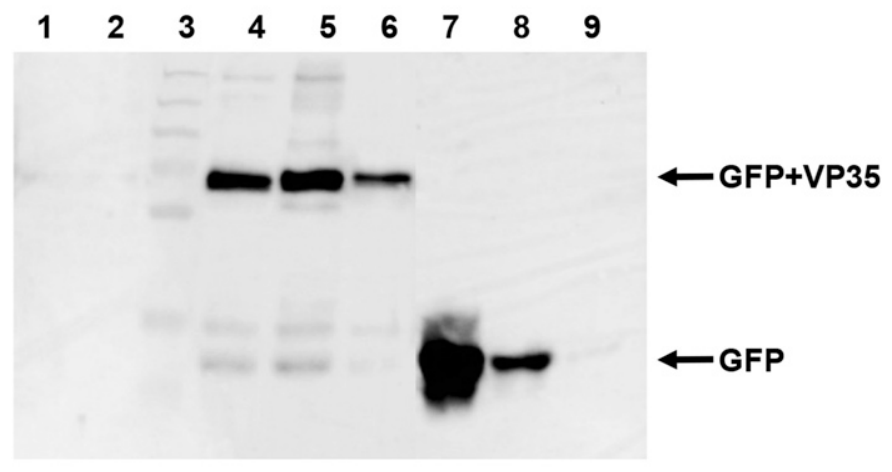

B

ToANV Antibody

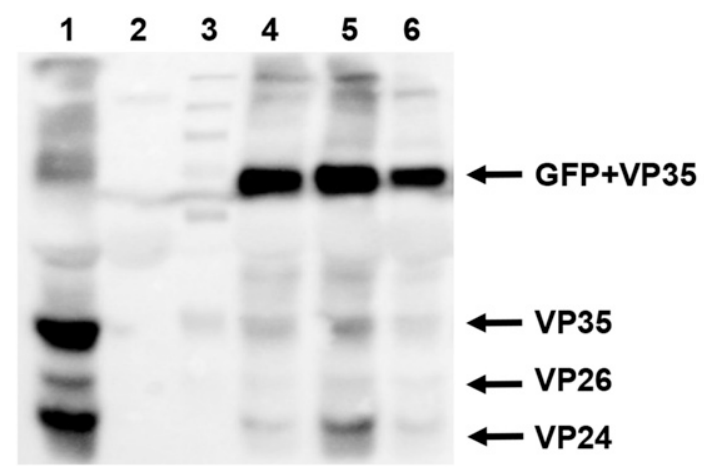

Fig. 4. Western blot analysis of Tomato marchitez virus isolate M (ToMarV-M)infected Nicotiana benthamiana plants. A, Western blot from leaves of $N$. benthamiana plants agroinfiltrated with ToMarV-M cDNA clone pJL89-M-R2GFP or pBIN-GFP 10-fold dilutions (1, 1:10, and 1:100) using green fluorescent protein (GFP) antibody. Lane 1, one $N$. benthamiana plant agroinfiltrated with ToMarV-M cDNA clones pJL89-M-R1 and pJL89-M-R2 in combination with pBIN-P19 and lane 2, one healthy $N$. benthamiana plant were used as negative controls; lane 3, Page ruler Protein Ladder (Thermo Fisher Scientific); lanes 4 to $6, N$. benthamiana plants infiltrated with cDNA clone pJL89-M-R2-GFP in combination with pJL89-M-R1 and pBIN-P19, 10-fold diluted 1 to 1:100, show the presence of GFP and the putative precursor (GFP+VP35); lanes 7 to 9, $N$. benthamiana plants infiltrated with only pBIN-GFP, 10-fold diluted 1 to 1:100 (positive control for GFP-antibody). Positions of putative GFP+VP35 precursor and GFP are indicated with an arrow. B, Western blot from leaves of $N$. benthamiana plants agroinfiltrated with ToMarV-M cDNA clone pJL89-M-R2-GFP or pBIN-GFP dilutions (1, 1:10, and 1:100) using Tomato apex necrosis virus (ToANV)-virion antibody. Lane $1, N$. benthamiana plants infiltrated with ToMarV-M cDNA clones pJL89-M-R1 and pJL89-M-R2 in combination with pBIN-P19 (positive control for ToANV-virion antibody); lane 2, one healthy $N$. benthamiana plant was used as negative control; lane 3, Page ruler Protein Ladder (Thermo Fisher Scientific); lanes 4 to $6, N$. benthamiana plants infiltrated with cDNA clone pJL89-M-R2-GFP in combination with pJL89-M-R1 and pBIN-P19, 10-fold diluted (1, 1:10, and 1:100), show the presence of the three coat proteins and the putative precursor (GFP+VP35). Positions of putative 62-kDa GFP+VP35 precursor and VP35, VP26, and VP24 capsid proteins are indicated by arrows. 
agroinfiltrated with the cDNA clone pJL89-M-R2-GFP, demonstrating that GFP is processed from the polyprotein. The site for processing of GFP is likely between amino acid positions 458 and 485 (KT756875) of the RNA2 ORF2 polyprotein of ToMarV-M, suggesting that the cleavage site between the MP and VP35 occurs upstream of amino acid 485. Nevertheless, proteins of higher molecular mass were also present; specifically, a high amount of a putative GFP+VP35 precursor was also present. We also wanted to determine whether the cDNA clone pJL89-M-R2-GFP accumulated each of the CP subunits: indeed, Western blot analysis for CP allowed us to detect the three proteins and a very abundant putative GFP+VP35 precursor (Fig. 4B).

To determine whether the cDNA clone pJL89-M-R2-GFP, when coinoculated with pJL89-M-R1, was able to give rise to virions, partial virion purification from the agroinfiltrated leaves was performed. At $2 \mathrm{dpa}$, virus particles could be seen by ISEM only in leaves agroinfiltrated with the wild type expressing ToMarV pJL89-M-R1 and pJL89-M-R2 cDNA clones but, at $3 \mathrm{dpa}$, leaves agroinfiltrated with pJL89-M-R2-GFP in combination with pJL89-M-R1 and pBIN-P19 showed, by TEM, the presence of virions with the same morphology as those derived from infections resulting from ToMarV-M pJL89-M-R1 and pJL89-M-R2 cDNA clones and the wild-type ToMarV-M (Supplementary Fig. S3). However, when the recombinant pJL89-M-R2-GFP was used, the amount of virions was less than for the wild-type virus.
We then wanted to test whether the recombinant pJL89-M-R2-GFP, when coinfiltrated with pJL89-M-R1, could give rise to infections that moved from cell to cell. We compared a time course of infection where dilution of the A. tumefaciens cultures ensured initial singlecell infections. As a control, we used a serial dilution of pBIN-GFP for GFP expression from a construct that does not move outside the initially transiently infected cell. Through serial dilution, we identified 1:5,000 as an A. tumefaciens culture dilution that allowed single-cell infection (data not shown). Observation of agroinfiltrated leaves $20 \mathrm{~h}$ postagroinfiltration showed a weak specific GFP expression in distantly scattered epidermal cells for both constructs (data not shown). Observation of the same areas 4 dpa showed that, in the pBIN-GFP construct, only single cells could be seen expressing GFP. However, when pJL89-M-R2-GFP was used along with pJL89-M-R1 at 4 dpa, GFP expression was visible in groups of cells. This indicated that the pJL89-M-R2-derived GFP was not localized to individual cells and cell-to-cell movement was occurring in the $N$. benthamiana epidermal leaves agroinfiltrated with pJL89-M-R2-GFP and pJL89-M-R1 (Fig. 5). To follow the infection development, we monitored the expression of GFP using a dissecting stereo microscope for 3 days every $12 \mathrm{~h}$ (Supplementary Fig. S4). As a final control, we coagroinfiltrated the wild-type virus pJL89-M-R1 and pJL89-M-R2 in combination with pBIN-GFP to exclude that the clusters of GFP-expressing cells shown in Figure 5 because the ToMarV pJL89-M-R2-GFP cDNA clones are derived not
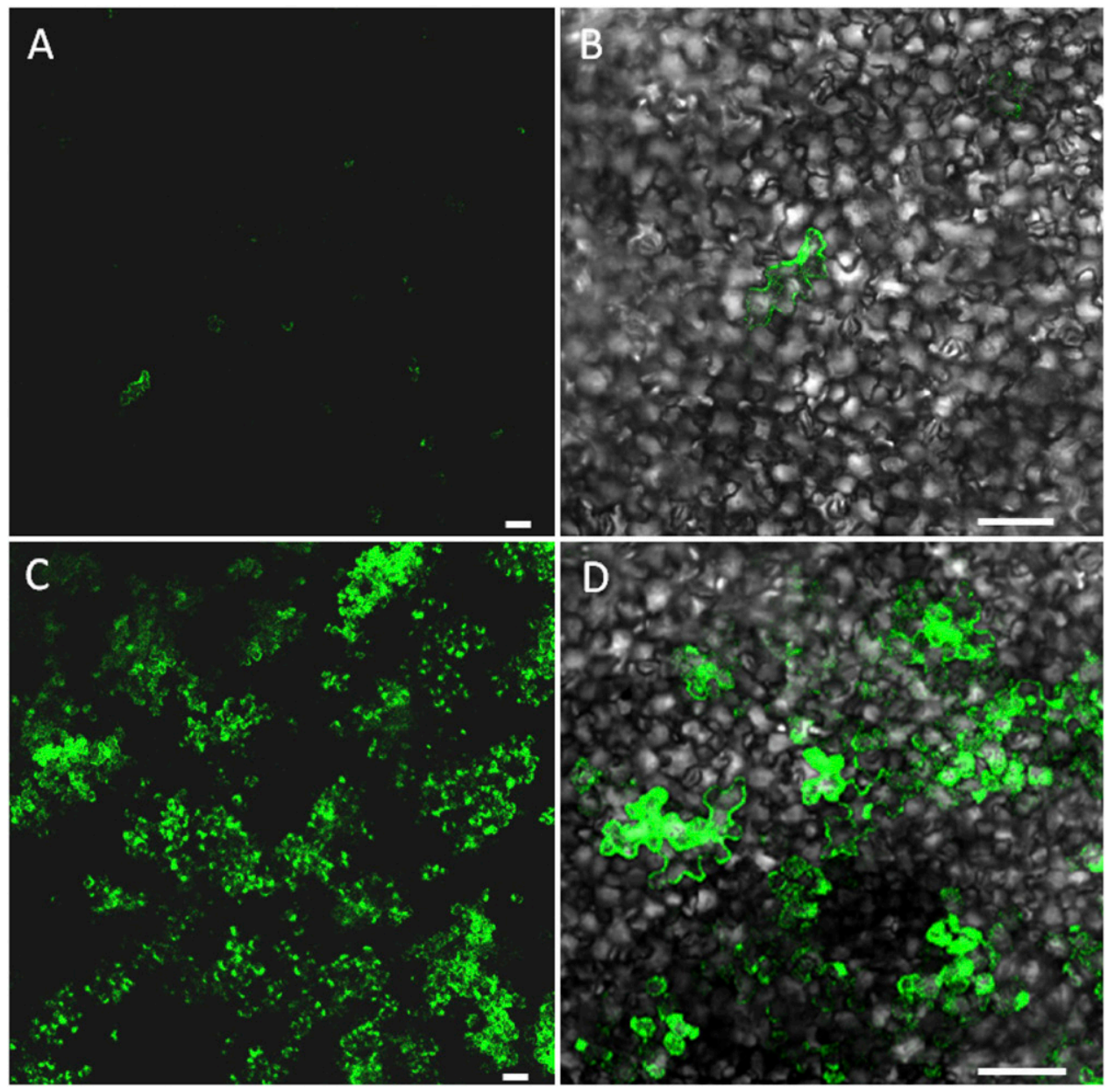

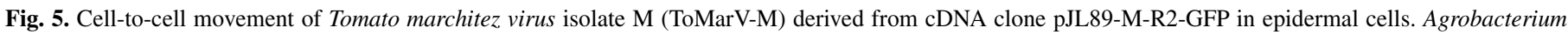

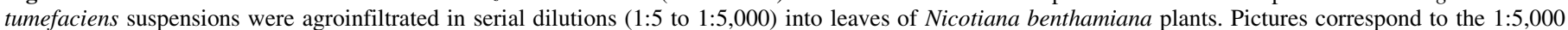

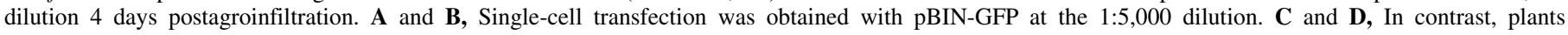

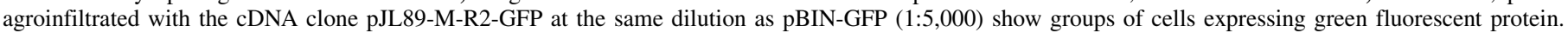

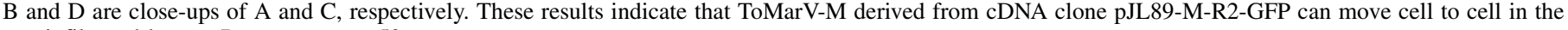
agroinfiltrated leaves. Bars represents $50 \mu \mathrm{m}$. 
TABLE 1. Whitefly transmission of ToMarV isolate M (ToMarV-M) derived from agroinfiltration of pJL89-M-R1 and pJL89-M-R2 cDNA clones using Bemisia tabaci biotype B

\begin{tabular}{llc}
\hline & \multicolumn{2}{c}{ Receptor plants $(\%)^{\mathrm{a}}$} \\
\cline { 2 - 3 } Source plant $^{\mathrm{b}}$ & Tomatillo & Tomato \\
\hline Tomato & $1 / 4(25)$ & $3 / 4(75)$ \\
Tomatillo & $4 / 4(100)$ & $4 / 4(100)$ \\
\hline
\end{tabular}

a Number of infected plants divided by the number of plants tested. Percentage of infection is indicated in parenthesis. Each experiment was only performed once.

b Plants showing systemic symptoms were used for transmission experiments. Approximately 1,000 whiteflies were allowed to acquire ToMarV-M from an infected tomato or tomatillo plant in a cage for $24 \mathrm{~h}$ before they were transferred to uninfected tomatillo or tomato plants.

TABLE 2. Screening of tomato lines for resistance to Tomato marchitez virus isolate $\mathrm{M}$ (ToMarV-M)

\begin{tabular}{lclll}
\hline & & \multicolumn{3}{c}{ Tomato lines $(\%)^{\mathrm{a}}$} \\
\cline { 3 - 5 } Inoculation method & Experiment $^{\mathrm{b}}$ & HMC101 & HMC102 & HMC103 \\
\hline Agroinfiltration $^{\mathrm{c}}$ & 1 & $9 / 9(100)$ & $0 / 9(0)$ & $0 / 9(0)$ \\
& 2 & $8 / 9(90)$ & $0 / 9(0)$ & $0 / 9(0)$ \\
Whitefly transmission $^{\mathrm{d}}$ & 1 & $7 / 10(70)$ & $0 / 10(0)$ & $0 / 10(0)$
\end{tabular}

a Number of infected plants divided by the number of plants tested at 15 days postagroinfiltration or posttransmission. Percentage of infection is indicated in parenthesis.

b Experiment replicate.

c Tomato plants were agroinfiltrated with equal amounts of ToMarV-M cDNA clones pJL89-M-R1 and pJL89-M-R2.

d Approximately 1,000 whiteflies were allowed to acquire ToMarV-M for $24 \mathrm{~h}$ in a cage before they were transferred to the uninfected tomato plants. from virus movement but from cell-to-cell GFP diffusion through enlarged plasmodesmata (Supplementary Fig. S5).

Western blot analysis of the dilution series above also showed that, when pBIN-GFP only was used, the amount of detectable protein decreased exponentially with the dilution series; whereas, in the case of the cDNA clone pJL89-M-R2-GFP and pJL89-M-R1, in the first three dilutions, no diminution of protein could be detected, providing further evidence of the fact that, when coinoculated with pJL89-M-R1, the cDNA clone pJL89-M-R2-GFP maintains high expression of GFP through newly infected cells (Figs. 4 and 5). However, systemic infection was not detected in the apical leaves of plants agroinfiltrated with pJL89-M-R1 and pJL89-M-R2-GFP 1 week postagroinfiltration, and this was confirmed by Western blot using GFP and ToANV-virion antibodies (data not shown).

The viral progeny derived from ToMarV-M cDNA clones is transmissible by B. tabaci biotype B. We next tested ToMarV$\mathrm{M}$-derived progeny resulting from agroinoculation for transmissibility by the whitefly vector $B$. tabaci biotype B. One tomato (line HMC101) and one tomatillo plant were each agroinfiltrated with the cDNA clones pJL89-M-R1 and pJL89-M-R2. When the plants showed systemic symptoms, they were exposed to B. tabaci biotype B for an AAP of $24 \mathrm{~h}$; then, the whiteflies were transferred to four tomato HMC101 and four tomatillo plants. ToMarV-M infection was scored by the development of specific symptoms (Fig. 2A). The progeny derived from the cDNA clones pJL89-M-R1 and pJL89-M-R2 were transmissible by $B$. tabaci biotype B to tomato HMC101 and tomatillo plants using both as source and receptor hosts. The percentage of transmission varied from 25 to $100 \%$ (Table 1).

Screening of tomato cultivars for resistance to ToMarV-M. We next assessed whether the ToMarV cDNA clones pJL89-M-R1 and pJL89-M-R2 could be used for screening tomato cultivars
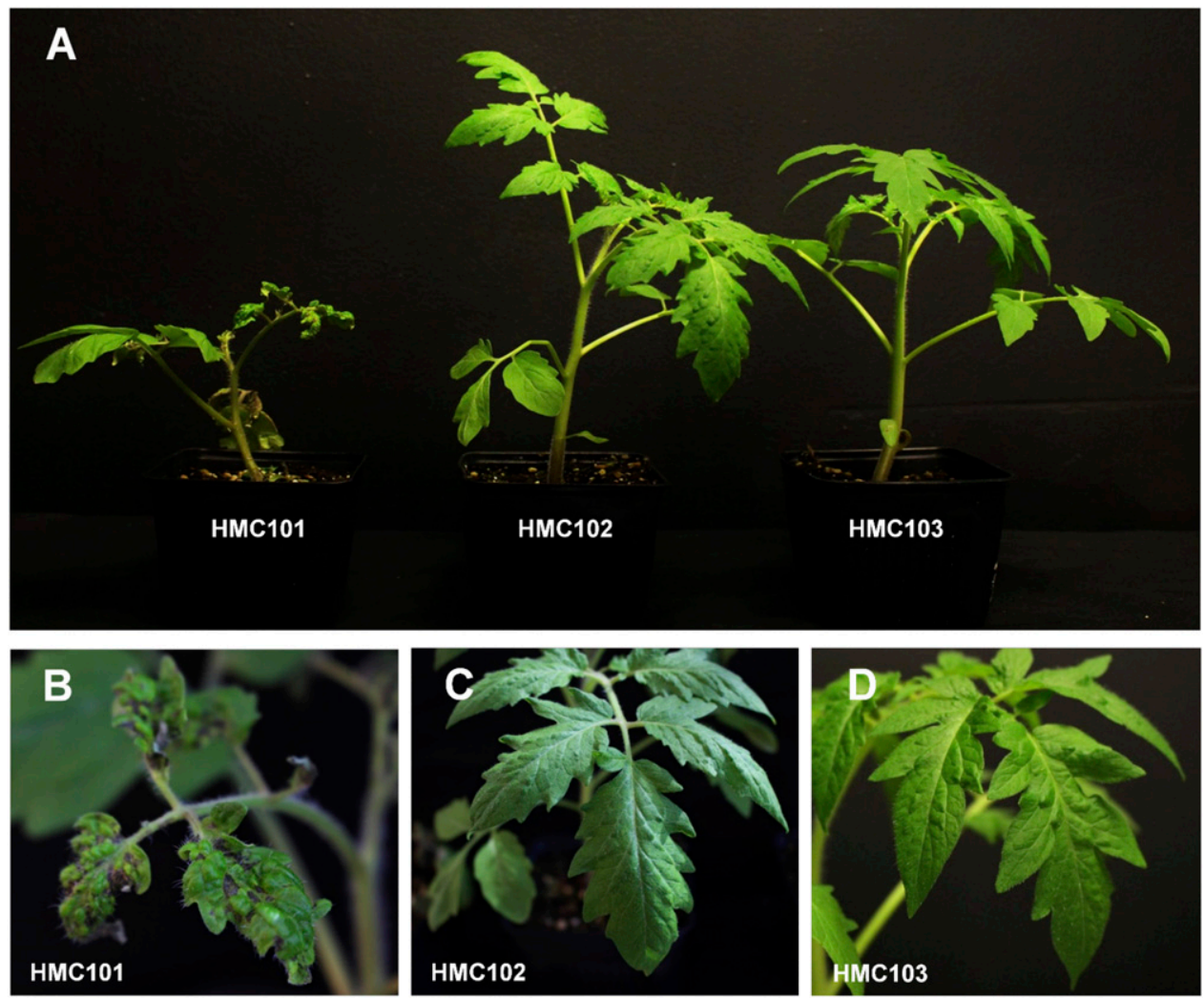

Fig. 6. Symptoms induced by Tomato marchitez virus isolate $M$ (ToMarV-M) derived from cDNA clones pJL89-M-R1 and pJL89-M-R2 at 15 days postagroinfiltration in plants of three tomato lines: HMC101, HMC102, and HMC103. A, Comparison of the three lines agroinfiltrated with pJL89-M-R1 and pJL89-M-R2. B, Detail of necrotic spots in upper noninoculated leaves of tomato HMC101 infected with pJL89-M-R1 and pJL89-M-R2. C and D, Lack of ToMarV-M symptoms in tomato lines HMC102 and HMC103 agroinfiltrated with pJL89-M-R1 and pJL89-M-R2. 
for resistance to ToMarV. This is of particular practical relevance because, unlike $N$. benthamiana and tomatillo plants, tomato plants are not efficiently infected with ToMarV by mechanical inoculation. Whitefly inoculation is relatively efficient but is cumbersome. Therefore, if we could directly agroinoculate tomato and obtain efficient infection in susceptible tomato, this would be desirable. We used three different tomato lines (HMC101, HMC102, and HMC103) and compared agroinfiltration of the cotyledons and whitefly transmission using B. tabaci. ToMarV-M clones were infectious in 90 to $100 \%$ of the susceptible HMC101 tomato plants whereas inoculation using B. tabaci gave only $70 \%$ infection (Table 2). The infected HMC101 tomato plants showed the typical symptoms of ToMarV-M (Fig. 6). None of the HMC102 and HMC103 tomato plants had symptoms of ToMarV-M when agroinfiltration or whitefly transmission were used for inoculation. These results indicated that the tomato lines HMC102 and HMC103 are resistant to ToMarV-M, whereas tomato line HMC101 is susceptible to ToMarV-M, and agroinfiltration is easier and equivalent or more efficient than is whitefly transmission for screening tomatoes for resistance to ToMarV.

\section{DISCUSSION}

Recently, ToMarV has been characterized as a new picorna-like virus belonging to the genus Torradovirus within the family Secoviridae (Sanfaçon et al. 2009; Turina et al. 2007). Previously, the full-length sequences of the gRNA of ToMarV isolate PRITMarV0601 and the partial sequences of the gRNA for ToANV isolate VE434 were determined (Turina et al. 2007; Verbeek et al. 2008). The consecutive discovery and reports of ToANV VE434 and ToMarV PRI-TMarV0601 during 2007 and 2008 (Turina et al. 2007; Verbeek et al. 2008), respectively, led to some confusion in the scientific community as to whether these two torradoviruses belonged to the same species and, therefore, should have the same name. The International Committee on Taxonomy of Viruses determined that both are the same species, with the name Tomato marchitez virus, and Tomato apex necrosis virus is a synonym of ToMarV (King et al. 2011). In the present work, the complete nucleotide sequences for ToMarV-M gRNA1 and -2 (from cDNA) were determined and used to construct full-length cDNA clones of ToMarV-M gRNAs. BLASTN analyses of ToMarV-M gRNA1 and -2 showed 99 and $98 \%$ nucleotide identity with the respective RNA for ToANV isolate VE434, and 95 and $80 \%$ nucleotide identity with ToMarV isolate PRI-TMarV0601, respectively. This suggests that all three isolates belong to the same species.

The 5'-terminal nucleotide is important for cDNA clone-derived infectivity in many plant viruses, including members of the family Secoviridae (Boyer and Haenni 1994; Eggen et al. 1989). The 5 -RACE of ToMarV-M revealed that the $5^{\prime}$-end sequences of both RNA1 and RNA2 are 5'-UUAAAAG- ${ }^{\prime}$. Also, the ToTV RNA1 $5^{\prime}$-end sequence starts with UUAAAAG but the RNA2 5' end begins with UUUAAAAG (Verbeek et al. 2007). The $5^{\prime}$ end of ToMarV-M and ToTV sequences are different from previously reported sequences for ToMarV isolate PRI-TMarV0601 that begin with AAAG at the $5^{\prime}$ termini of both RNA1 and RNA2 (Verbeek et al. 2008). Tomato chocolate spot virus and Tomato chocolate virus 5' ends begin with AAAAG (Batuman et al. 2010; Verbeek et al. 2010). Differences in the $5^{\prime}$ termini of different isolates of the same species have been reported among other members of the family Secoviridae such as the fabavirus Broad bean wilt virus 2 (Atsumi et al. 2013) and other plant viruses (Stewart et al. 2012). Whether the reported differences observed at the gRNA $5^{\prime}$ ends of tomato-infecting torradoviruses reflect authentic variation or sequencing artifacts remains to be determined.

In this work, we reported the first infectious clone of ToMarV and the second infectious clone of a torradovirus, ToTV (Wieczorek et al. 2015). The biological activity of the progeny virus derived from the cDNA clones pJL89-M-R1 and pJL89-M-R2 was comparable with that of the wild-type virus. This system triggered symptoms typical of ToMarV-M infection in tomato plants. Interestingly, it also produced systemic infection in the model plant $N$. benthamiana and in tomatillo plants, the latter being a suitable host for whitefly vector transmission studies (Fig. 2A). Purified virions showed the presence of spherical particles (Fig. 2C) and RNA extracted from these particles confirmed the presence of both gRNAs (Fig. 2D).

Virus transmission assays with $B$. tabaci biotype B demonstrated that the viral progeny derived from the cDNA clones pJL89-M-R1 and pJL89-M-R2 was transmissible to tomatillo and tomato plants (Tables 1 and 2). Whiteflies in the genera Bemisia and Trialeurodes are both reported as vectors for torradoviruses. These are ubiquitous in Mexico, and our data are the first report of whitefly transmission of a torradovirus derived from cDNA infectious clones. We have opened a research path for studying the mode and molecular determinants of the whitefly transmission of torradoviruses.

Control of tomato-infecting torradoviruses such as ToTV has relied on the use of different methods: early eradication of foci of infection, control of the vector, and the use of genetic resistance (Gómez et al. 2012). Genetic resistance has been used for the sustainable control of many plant virus diseases (Gómez et al. 2009) and is desirable for ToMarV. ToMarV is inefficiently transmitted to tomato mechanically; therefore, the use of an agroinfiltration system to inoculate tomato with ToMarV-M may facilitate the discovery of new resistance sources and identify tomato lines resistant to ToMarV. These results highlight the potential use of a ToMarV-M agroinoculation system to screen for resistance against ToMarV.

The family Secoviridae includes the genera Comovirus, Nepovirus, Fabavirus, Sequivirus, Waikavirus, Cheravirus, Sadwavirus, and Torradovirus. These viruses have monopartite or bipartite genomes and use a polyprotein strategy to express their proteins (Sanfaçon et al. 2009; van der Vlugt 2015). Little is known about the functions of torradovirus proteins or their replication and polyprotein strategies. In this work, we demonstrated that RNA1 of the torradovirus ToMarV-M is required for the replication of RNA2 and can replicate independently from RNA2. The same replication strategy is used for other bipartite members of the family Secoviridae, such as members of the genera Nepovirus and Comovirus (Goldbach et al. 1980; Robinson et al. 1980). However, this is the first demonstration for torradoviruses. Also, we developed a system for the expression of GFP in cis by inserting its ORF between the putative MP of ToMarV-M and the CP VP35. When coinoculated with pJL89-M-R1, the cDNA clone pJL89-M-R2-GFP expressed the GFP and ToMarV-M CP in agroinfiltrated leaves but there was no detectable systemic infection in the upper, noninoculated leaves. Western blot analysis indicated that the GFP was processed from the RNA2-encoded polyprotein of the cDNA clone pJL89-M-R2-GFP. The GFP was placed between amino acid 458 and 485 of the RNA2 ORF2 polyprotein. Thus, the cleavage site between the MP and the VP35 CP might be located upstream of amino acid position 485, as occurs in other torradoviruses such as ToTV (Verbeek et al. 2007). The presence of a high amount of GFP+VP35 precursor in the Western blot analysis suggests that the processing and release of GFP from the polyprotein by the protease might not be very efficient. Moreover, this could explain why the cDNA clone pJL89-M-R2-GFP plus pJL89-M-R1 was not able to trigger systemic infection.

We confirmed the presence of ToMarV-M virions in leaves agroinfiltrated with pJL89-M-R2-GFP and pJL89-M-R1, although the delay, when compared with the wild-type, does not allow us to rule out the hypothesis that such virions derived from a small amount of recombination occurring during replication of the viral vector. Our data may suggest that, because of inefficient processing, we may have fewer virions and less cell-to-cell movement when the cDNA clone pJL89-M-R2-GFP was used. Differences in the length of the duplicated sequences of the cleavage site between the 
$\mathrm{MP}$ and the large coat protein of Cowpea mosaic virus (CPMV) determined the systemic infection of CPMV (Gopinath et al. 2000; Zhang and Ghabrial 2006). The cleavage sites of ToMarV-M RNA2-encoded polyprotein remain to be determined and will allow us to develop a system that might trigger systemic infection. Although the infection with the cDNA clone ToMarV-M pJL89M-R2-GFP was not systemic, cell-to-cell movement of the pJL89M-R2-GFP viral progeny in the epidermal cells of $N$. benthamiana was observed. Therefore, our system has an important application as a reverse genetic tool for gene function studies of the viral components involved in the cell-to-cell movement.

Members of the genus Torradovirus are newly emergent plant viruses that infect different hosts such as tomato, carrot, cassava, motherwort, and lettuce (van der Vlugt 2015). The molecular biology, epidemiology, and evolution of ToMarV has not yet been studied in depth. The use of full-length cDNA clones of ToMarV-M that are infectious in tomato and in the model plant $N$. benthamiana will allow us to extend our knowledge of different aspects of the molecular biology of torradoviruses and to identify new sources of genetic resistance to control of ToMarV.

\section{ACKNOWLEDGMENTS}

This research was partially funded by grant SCB11058 from the California Department of Food and Agriculture. M. Turina and E. ZamoraMacorra were supported by a short-term mobility scholarship of the Italian CNR and the Mexican government (Consejo Nacional de Ciencia y Tecnología, CONACYT), respectively. We thank T. Tian for his help with the TEM, M. Vallino for helping with the confocal microscope, D. Baulcombe for kindly providing plasmid pBIN-P19, and E. J. Vivoda from H\&M Clause (Davis, CA) for providing the tomato lines used in this work.

\section{LITERATURE CITED}

Altschul, S. F., Gish, W., Miller, W., Myers, E. W., and Lipman, D. J. 1990. Basic local alignment search tool. J. Mol. Biol. 215:403-410.

Atsumi, G., Tomita, R., Kobayashi, K., and Sekine, K.-T. 2013. Establishment of an agroinoculation system for Broad bean wilt virus 2. Arch. Virol. 158: 1549-1554.

Barajas-Ortiz, M., León-Sicairos, C., López-Valenzuela, J., Reyes-Moreno, C., Valdez-Ortiz, A., Velarde-Félix, S., Peraza-Garay, F., and Garzón-Tiznado, J. 2013. Transmission efficiency of Tomato apex necrosis virus by Bemisia tabaci (Hemiptera: Aleyrodidae) biotype B in tomato. J. Econ. Entomol. 106:1559-1565.

Batuman, O., Kuo, Y.-W., Palmieri, M., Rojas, M., and Gilbertson, R. 2010. Tomato chocolate spot virus, a member of a new Torradovirus species that causes a necrosis-associated disease of tomato in Guatemala. Arch. Virol. 155:857-869.

Bonfield, J. K., Smith, K. F., and Staden, R. 1995. A new DNA sequence assembly program. Nucleic Acids Res. 23:4992-4999.

Boyer, J.-C., and Haenni, A.-L. 1994. Infectious transcripts and cDNA clones of RNA viruses. Virology 198:415-426.

Crivelli, G., Ciuffo, M., Genre, A., Masenga, V., and Turina, M. 2011. Reverse genetic analysis of Ourmiaviruses reveals the nucleolar localization of the coat protein in Nicotiana benthamiana and unusual requirements for virion formation. J. Virol. 85:5091-5104.

Eggen, R., Verver, J., Wellink, J., De Jong, A., Goldbach, R., and van Kammen, A. 1989. Improvements of the infectivity of in vitro transcripts from cloned cowpea mosaic virus cDNA: Impact of terminal nucleotide sequences. Virology 173:447-455.

Goldbach, R., Rezelman, G., and van Kammen, A. 1980. Independent replication and expression of B-component RNA of cowpea mosaic virus. Nature 286:297-300.
Gómez, P., Rodríguez-Hernández, A., Moury, B., and Aranda, M. 2009. Genetic resistance for the sustainable control of plant virus diseases: Breeding, mechanisms and durability. Eur. J. Plant Pathol. 125:1-22.

Gómez, P., Sempere, R., and Aranda, M. A. 2012. Pepino mosaic virus and Tomato torrado virus: Two emerging viruses affecting tomato crops in the Mediterranean Basin. Pages 505-532 in: Advances in Virus Research, Vol. 84. L. Gad and L. Hervé, eds. Academic Press, London.

Gopinath, K., Wellink, J., Porta, C., Taylor, K. M., Lomonossoff, G. P., and van Kammen, A. 2000. Engineering Cowpea mosaic virus RNA-2 into a vector to express heterologous proteins in plants. Virology 267:159-173.

King, A. M., Adams, M. J., and Lefkowitz, E. J., eds. 2011. Virus Taxonomy: Classification and Nomenclature of Viruses. Ninth Report of the International Committee on Taxonomy of Viruses. Elsevier, London.

Komatsu, K., Hashimoto, M., Okano, Y., Keima, T., Kitazawa, Y., Nijo, T., Takahashi, S., Maejima, K., Yamaji, Y., and Namba, S. 2013. Construction of an infectious cDNA clone of Radish mosaic virus, a crucifer-infecting comovirus. Arch. Virol. 158:1579-1582.

Lorang, J., Tuori, R., Martinez, J., Sawyer, T., Redman, R., Rollins, J., Wolpert, T., Johnson, K., Rodriguez, R., and Dickman, M. 2001. Green fluorescent protein is lighting up fungal biology. Appl. Environ. Microbiol. 67:1987-1994.

Robinson, D., Barker, H., Harrison, B., and Mayo, M. 1980. Replication of RNA-1 of tomato black ring virus independently of RNA-2. J. Gen. Virol. 51:317-326

Rossi, M., Vallino, M., Abbà, S., Ciuffo, M., Balestrini, R., Genre, A., and Turina, M. 2015. The importance of the KR-Rich region of the coat protein of Ourmia melon virus for host specificity, tissue tropism, and interference with antiviral defense. Mol. Plant-Microbe Interact. 28:30-41.

Sambrook, J., and Russell David, W. 1989. Molecular Cloning: A Laboratory Manual, Vol. 3. Cold Spring Harbor Laboratory Press, Cold Spring Harbor, NY.

Sanfaçon, H., Wellink, J., Le Gall, O., Karasev, A., Van der Vlugt, R., and Wetzel, T. 2009. Secoviridae: A proposed family of plant viruses within the order Picornavirales that combines the families Sequiviridae and Comoviridae, the unassigned genera Cheravirus and Sadwavirus, and the proposed genus Torradovirus. Arch. Virol. 154:899-907.

Stewart, L., Bouchard, R., Redinbaugh, M., and Meulia, T. 2012. Complete sequence and development of a full-length infectious clone of an Ohio isolate of Maize dwarf mosaic virus (MDMV). Virus Res. 165:219-224.

Tamura, K., Stecher, G., Peterson, D., Filipski, A., and Kumar, S. 2013. MEGA6: Molecular Evolutionary Genetics Analysis version 6.0. Mol. Biol. Evol. 30:2725-2729.

Turina, M., Ricker, M., Lenzi, R., Masenga, V., and Ciuffo, M. 2007. A severe disease of tomato in the Culiacan area (Sinaloa, Mexico) is caused by a new picorna-like viral species. Plant Dis. 91:932-941.

van der Vlugt, R. 2015. Torradoviruses. Annu. Rev. Phytopathol. 53:485-512.

Verbeek, M., Dullemans, A., van den Heuvel, J., Maris, P., and Van der Vlugt, R. 2007. Identification and characterisation of Tomato torrado virus, a new plant picorna-like virus from tomato. Arch. Virol. 152:881-890.

Verbeek, M., Dullemans, A., van den Heuvel, J., Maris, P., and Van der Vlugt, R. 2008. Tomato marchitez virus, a new plant picorna-like virus from tomato related to Tomato torrado virus. Arch. Virol. 153:127-134.

Verbeek, M., Dullemans, A., van den Heuvel, H., Maris, P., and van der Vlugt, R. 2010. Tomato chocolate virus: A new plant virus infecting tomato and a proposed member of the genus Torradovirus. Arch. Virol. 155:751755.

Verbeek, M., van Bekkum, P. J., Dullemans, A. M., and van der Vlugt, R. A. 2014. Torradoviruses are transmitted in a semi-persistent and stylet-borne manner by three whitefly vectors. Virus Res. 186:55-60.

Wang, J., Turina, M., Stewart, L. R., Lindbo, J. A., and Falk, B. W. 2009. Agroinoculation of the Crinivirus, Lettuce infectious yellows virus, for systemic plant infection. Virology 392:131-136.

Wieczorek, P., Budziszewska, M., and Obrępalska-Stęplowska, A. 2015. Construction of infectious clones of Tomato torrado virus and their delivery by agroinfiltration. Arch. Virol. 160:517-521.

Zhang, C., and Ghabrial, S. A. 2006. Development of Bean pod mottle virusbased vectors for stable protein expression and sequence-specific virusinduced gene silencing in soybean. Virology 344:401-411. 